\title{
Direct proximal right subclavian artery cannulation during surgery of the thoracic aorta
}

Tommaso Regesta, MD, ${ }^{\text {a }}$ Corrado Cavozza, MD, ${ }^{\text {a }}$ Antonio Campanella, MD, ${ }^{\mathrm{a}}$ Pasquale Pellegrino, MD, ${ }^{\mathrm{a}}$ Riccardo Gherli, MD, ${ }^{a}$ Giulia Maj, MD, ${ }^{b}$ and Andrea Audo, MD $^{\mathrm{a}}$

\section{ABSTRACT}

Objective: To evaluate outcomes of single sternum access for right subclavian artery cannulation without infraclavicular incision in surgery of the thoracic aorta.

Methods: Between January 2015 and December 2019, 44 consecutive patients underwent surgery of the thoracic aorta with cannulation of the right subclavian artery, after sternotomy and before pericardiotomy, through a direct percutaneous cannula with a single access without additional infraclavicular skin incision. The indication for surgery was type A acute aortic dissection in 29 patients $(65.9 \%)$, proximal aortic aneurysm in $11(25 \%)$, and aneurysm of the aortic arch in $4(9 \%)$. Operative procedures were replacement of the ascending aorta in 23 patients, Bentall procedure in 10, hemiarch replacement in 6, and total arch replacement in 5. The mean cardiopulmonary bypass (CPB) and cross-clamp times were $185 \pm 62$ minutes and $138 \pm 41$ minutes, respectively.

Results: The in-hospital mortality rate was $6.8 \%$. Permanent neurologic dysfunction occurred in 3 patients $(6.8 \%)$ and temporary neurologic dysfunction occurred in 4 patients $(9.0 \%)$. There were no vascular complications related to this technique. No lesions to the vagus and recurrent laryngeal nerves have been reported.

Conclusions: In our experience, a single sternum access for right subclavian artery cannulation avoids the risk and complications of an infraclavicular incision required for axillary artery cannulation. This technique is safe and represent a valid option for $\mathrm{CBP}$ and antegrade cerebral perfusion during surgery of the thoracic aorta. (JTCVS Techniques 2021;8:1-6)

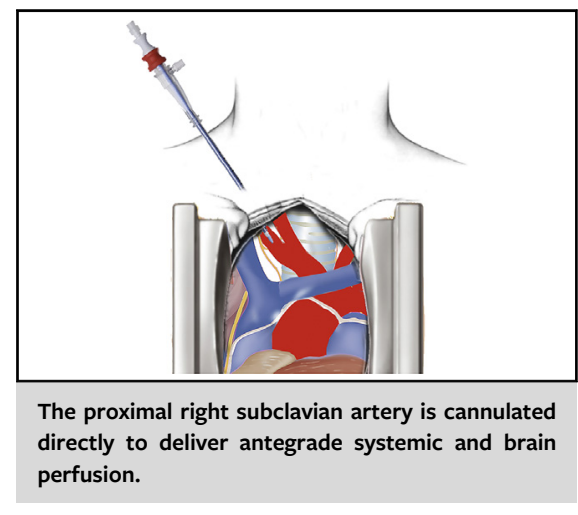

CENTRAL MESSAGE

Direct percutaneous proximal

right subclavian artery cannula-

tion without any other incision

than median sternotomy for

thoracic aortic surgery is safe

and yields excellent results.

\section{PERSPECTIVE}

We describe an alternative technique of percutaneous direct proximal right subclavian artery cannulation for delivering antegrade systemic and brain perfusion during thoracic aorta surgery. This technique eliminates the risk associated with axillary artery cannulation and yields excellent postoperative outcomes.

See Commentaries on pages 7 and 9.
From the Departments of ${ }^{\mathrm{a}}$ Cardiac Surgery and ${ }^{\mathrm{b}}$ Anesthesia and Intensive Care, Azienda Ospedaliera SS Antonio e Biagio e Cesare Arrigo, Alessandria, Italy. Drs Regesta and Cavozza contributed equally to this work and should be considered co-first authors.

Received for publication April 17, 2021; accepted for publication April 20, 2021; available ahead of print April 27, 2021

Address for reprints: Corrado Cavozza, MD, Cardiac Surgery, ASO SS Antonio e Biagio, Via Venezia 16, 15121 Alessandria, Italy (E-mail: cc220564@gmail.com). 2666-2507

Copyright $\odot 2021$ Published by Elsevier Inc. on behalf of The American Association for Thoracic Surgery. This is an open access article under the CC BY-NC-ND license (http://creativecommons.org/licenses/by-nc-nd/4.0/).

https://doi.org/10.1016/j.xjtc.2021.04.017
Controversy remains regarding the best cannulation site and cerebral perfusion strategy for surgery of the thoracic aorta. Femoral artery cannulation is simple and fast; however, it carries a risk of retrograde thrombotic embolism and requires further strategies for cerebral protection, which can increase the complexity of the operation. ${ }^{1}$ Innominate artery (IA) cannulation provides excellent results, ${ }^{2}$ but the IA is often involved in the dissection process and thus cannot be used safely during surgery for type A aortic 


\section{Abbreviations and Acronyms}

$\mathrm{ACP}=$ antegrade cerebral perfusion

$\mathrm{CPB}=$ cardiopulmonary bypass

IA $=$ innominate artery

$\mathrm{TAAD}=$ thoracic aortic aneurysm dissection

dissection. Axillary artery cannulation is a widely adopted technique to establish cardiopulmonary bypass (CPB) and achieve cerebral protection; however, it requires a separate infraclavicular incision and carries a high risk of complications. $^{3}$

A single sternum access surgical incision with extension to the right neck for direct cannulation of the right subclavian artery avoids the need for an additional infraclavicular incision access and can be safely used as site for CBP and antegrade unilateral cerebral perfusion. In this study, we present our experience with 44 patients who underwent surgery of the thoracic aorta with this technique.

All patients (and/or relatives) provided consent for the use of personal information. This study was conducted in accordance with the ethical principles established in the Declaration of Helsinki and approved by the hospital's Institutional Review Board.

\section{METHODS}

This series comprised 44 consecutive patients who underwent surgery of the thoracic aorta and received a single sternum single surgical incision extended to the right neck access for direct right subclavian artery cannulation as a site for CBP and antegrade cerebral perfusion (ACP) between January 2015 and December 2019. The mean patient age was $72 \pm 12$ years, and 29 were male $(66 \%)$. Patient characteristics are summarized in Table 1.

\section{Surgical Technique}

The operative technique has been described previously. ${ }^{4}$ In brief, all patients underwent full median sternotomy, and the skin incision was extended to the right neck. Before pericardial opening, the innominate

TABLE 1. Patient characteristics

\begin{tabular}{|c|c|}
\hline Characteristic & Value \\
\hline Age, $y$, mean \pm SD & $72 \pm 12$ \\
\hline Male sex, n (\%) & $29(66)$ \\
\hline NYHA class $\geq 3, \mathrm{n}(\%)$ & $7(16)$ \\
\hline Diabetes mellitus, n (\%) & $9(20)$ \\
\hline Hypertension, $\mathrm{n}(\%)$ & $32(73)$ \\
\hline Renal failure, $\mathrm{n}(\%)$ & $11(25)$ \\
\hline COPD, n $(\%)$ & $3(7)$ \\
\hline Recent stroke, $\mathrm{n}(\%)$ & $2(5)$ \\
\hline Aortic dissection, $\mathrm{n}(\%)$ & $29(66)$ \\
\hline Thoracic aortic aneurysm, $\mathrm{n}(\%)$ & $15(34)$ \\
\hline
\end{tabular}

vein is identified and retracted inferiorly with an umbilical tape. The IA is exposed, and the proximal part of the subclavian artery is isolated. Care must be taken to avoid injury to the vagus nerve that crosses the first part of the lower margin of the subclavian artery anteriorly (Figure 1). After heparinization, the subclavian artery is cannulated directly through a skin cutdown (Figures 2 and 3). Usually a 20 or 18 Fr OptiSite arterial cannula (Edwards Lifesciences, Irvine, Calif) is inserted using the Seldinger technique (Video 1). Once CPB is established, the operation is performed in the usual manner.

We routinely cool the core temperature to $26^{\circ} \mathrm{C}$ and provide unilateral ACP through the right subclavian artery at a target flow of $10 \mathrm{~mL} / \mathrm{kg}$ to protect the brain. Brain perfusion is assessed with near-infrared spectroscopy capillary saturation; if a unilateral drop in saturation to $<20 \%$ of the baseline value is observed, bilateral perfusion through a second cannula inserted in the left common carotid artery is performed. We routinely perform bilateral cerebral perfusion in cases of total arch replacement. An adequate pump flow rate was always achieved without concern for high pressure in the arterial line. Myocardial protection was achieved with crystalloid cold cardioplegia (Custodiol HTK; Essential Pharmaceuticals, Durham, $\mathrm{NC}$ ).

Mean and range or mean \pm standard deviation are used to describe continuous data. Categorical variables are expressed as percentages. The types of operations performed and perfusion data are provided in Table 2.

\section{RESULTS}

Three patients (6.8\%) experienced in-hospital mortality, all of whom were operated on for type A thoracic acute

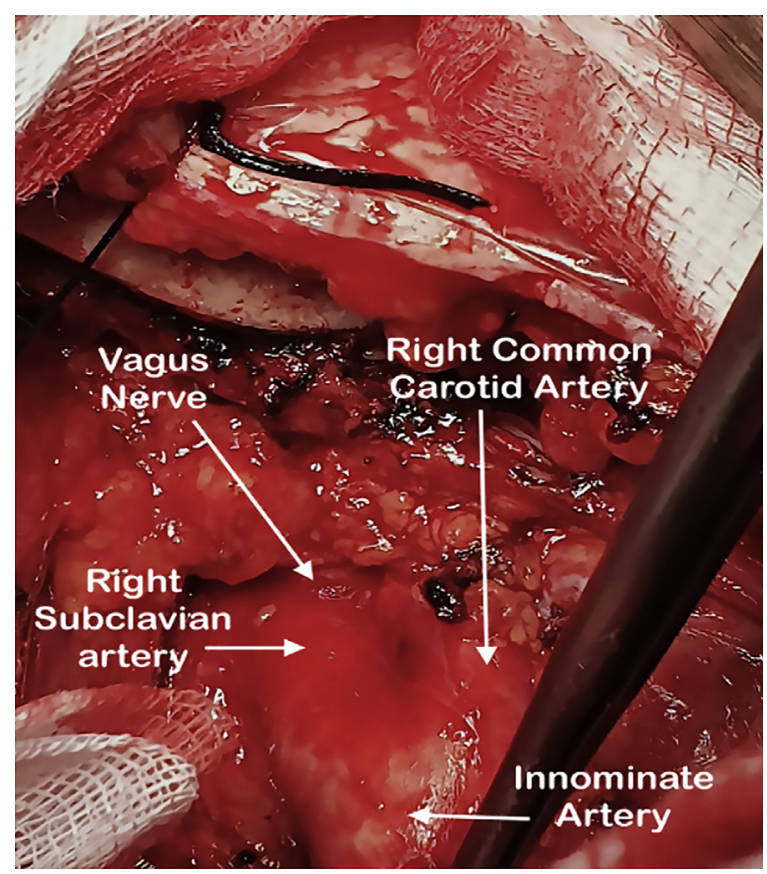

FIGURE 1. The median sternotomy is extended to the right neck. The innominate artery (IA) is exposed and dissected up to its bifurcation and the origin of the right subclavian artery (RSCA). The first part of the RSCA arises from the bifurcation of the IA, deep to the sternocostal joint to the medial edge of the scalenus anterior muscle. The vagus nerve descends within the carotid sheath, between the internal jugular vein and the internal and right common carotid artery, then crosses the RSCA anteriorly. Care must be taken to avoid injuring the vagus nerve. 


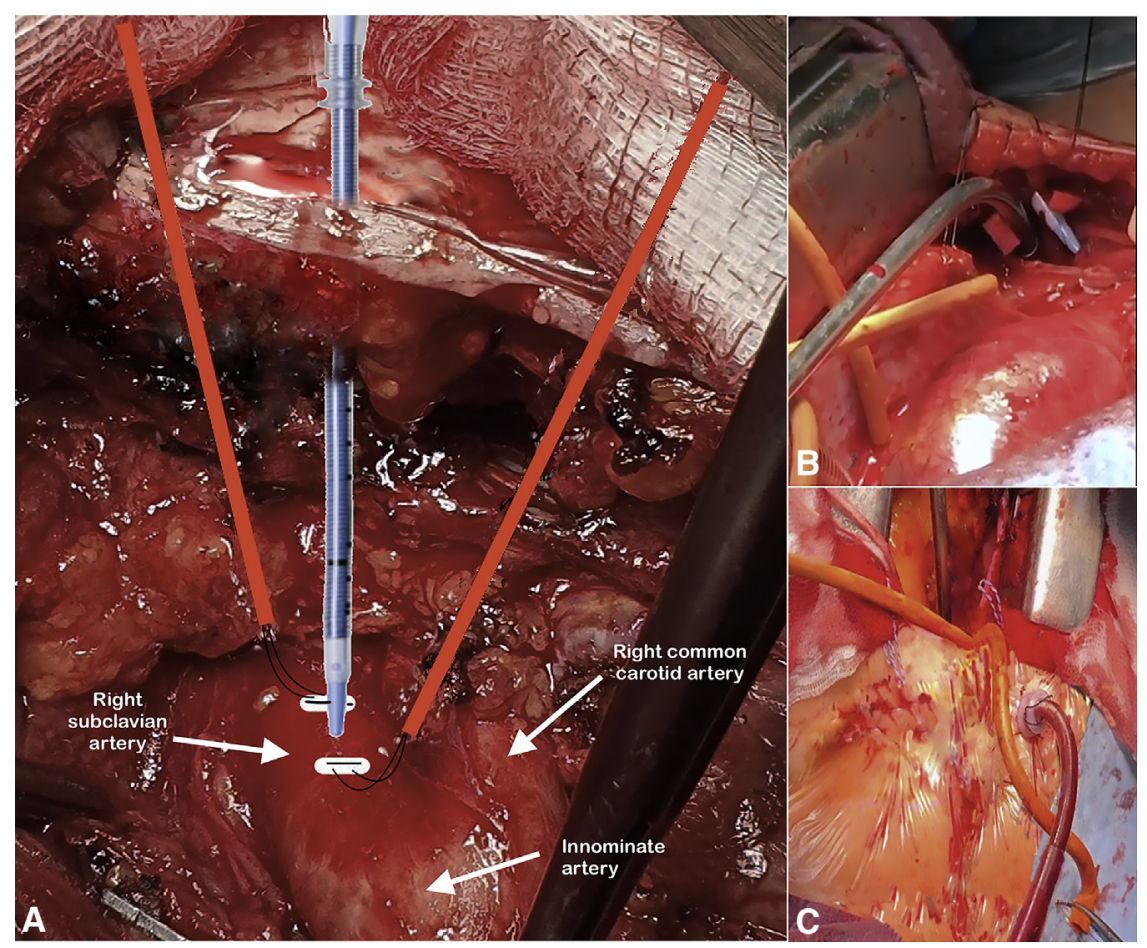

FIGURE 2. A, After heparinization, 2 double-pledget stitches (polypropylene 4/0) with tourniquets are placed on the proximal portion of the right subclavian artery. An OptiSite arterial cannula is inserted directly through a skin cutdown, via the Seldinger technique. The cannula is carefully advanced into the artery to avoid damage of the vessel. B, Adequate tissue dilatation before cannulation is key to aid cannula insertion. C, A cannula inserted through a skin incision without any other incision than median sternotomy.

aortic dissection (TAAD). The cause of death was low cardiac output syndrome in 2 patients and sepsis in 1 patient. Permanent neurologic dysfunction occurred in 3 patients $(6.8 \%)$. Two of these patients presented with preoperative neurologic dysfunction due to TAAD, and 1 patient

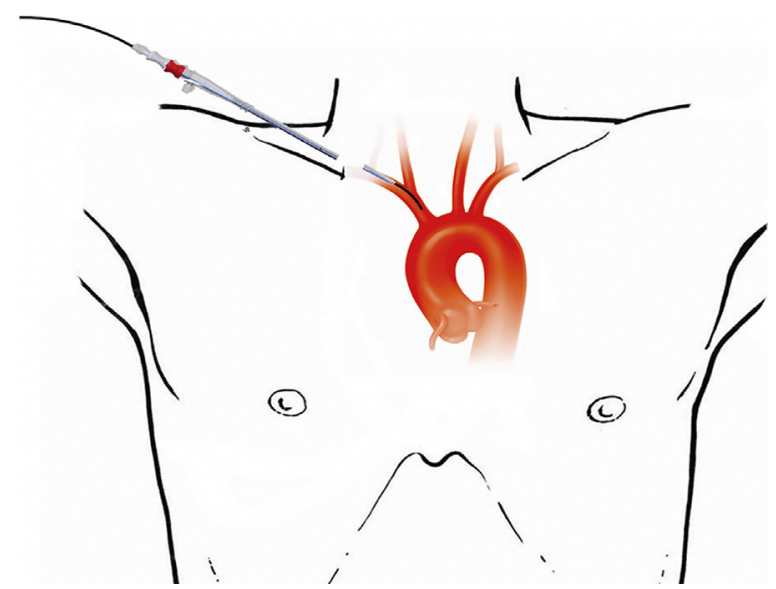

FIGURE 3. Direct proximal right subclavian artery cannulation is performed via the Seldinger technique through a skin incision made $2 \mathrm{~cm}$ lateral to the upper part of the sternotomy. The guide wire should be introduced very carefully to avoid tearing the intima. The tip of the cannula should not be advanced more than $2 \mathrm{~cm}$ in the artery to allow safe crossclamping of the innominate artery during circulatory arrest. underwent redo surgery with total arch replacement using the frozen elephant trunk technique (Thoraflex; Vascutek, Terumo, Inchinnan, Scotland, United Kingdom) and developed paraplegia. Temporary neurologic dysfunction, defined as postoperative delirium, agitation, and transient motor deficit, occurred in 4 patients $(9.1 \%)$ (Figure 4 ). Computed tomography scan of the brain did not reveal any neurologic damage, and all 4 patients were discharged without symptoms. Two patients $(4.5 \%)$ required tracheostomy because of postoperative respiratory insufficiency, and 2 patients $(4.5 \%)$ required permanent hemodialysis. Three patients $(6.8 \%)$ underwent reexploration for surgical bleeding. Postoperative outcomes are listed in Table 3.

\section{DISCUSSION}

Surgery of the thoracic aorta is challenging, with significant rates of complications. ${ }^{5}$ One of the main concerns is the choice of arterial cannulation site and cerebral perfusion strategy. To achieve optimal cerebral protection during aortic surgery, the risk of embolization of the brain and organ malperfusion should be reduced by avoiding retrograde perfusion strategies. ${ }^{1,6}$ To further improve neurologic outcomes, brain perfusion with ACP during the circulatory arrest period is mandatory. Ideally, the best theoretical option is to establish CPB and ACP using the same arterial cannula. If unilateral brain perfusion can be safely performed, 


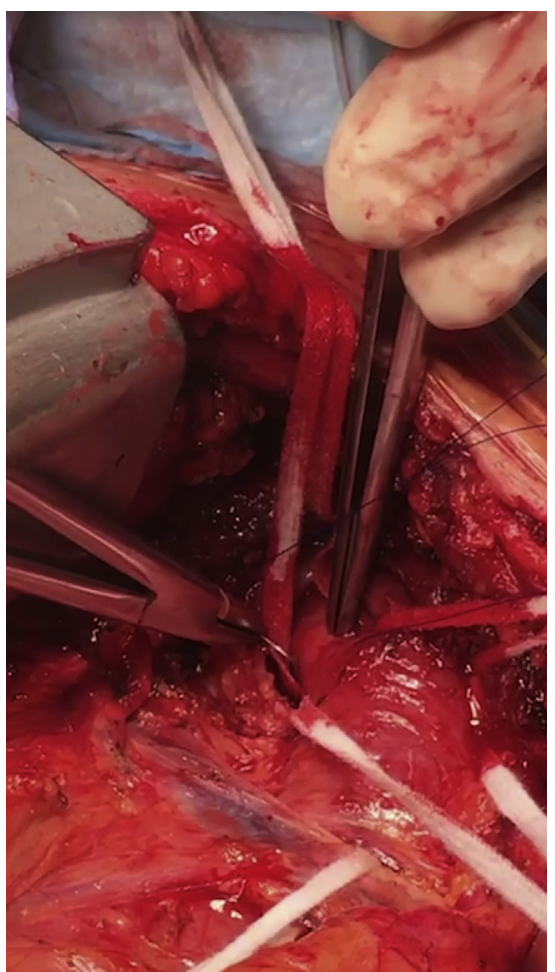

VIDEO 1. After a median sternotomy, the skin incision is extended to the right neck. Before pericardial opening, the innominate vein is isolated and retracted with an umbilical tape to facilitate dissection of the supra-aortic vessels. The innominate artery and the right subclavian artery are also isolated and taped. Care must be taken to avoid injury to the vagus nerve. After heparinization, 2 double-pledget stitches (polypropylene 4/0) with tourniquets are placed on the proximal portion of the right subclavian artery. An OptiSite arterial cannula is inserted directly through a skin cutdown using the Seldinger technique. Adequate tissue dilatation before cannulation is key to aid cannula insertion. The cannula must be carefully inserted, and the tip should not be advanced more than $2 \mathrm{~cm}$. Cardiopulmonary bypass is started, and surgery is performed in the usual fashion.Video available at: https://www.jtcvs.org/article/S2666-2507(21)00312-6/fulltext.

this can be achieved by cannulating the right axillary artery or the IA; however, when bilateral perfusion is necessary, a cannula must be placed in the left carotid artery.

Cannulation of the right axillary artery is a widely adopted technique to establish CPB and unilateral ACP. Two different technique have been described: direct cannulation and cannulation through a side graft sewn to the artery, always with a separate infraclavicular incision, which has been associated with significant complications. ${ }^{3}$

Cannulation of the IA during surgery of the thoracic aorta provides good results ${ }^{4}$ and offers several advantages. It does not require a second surgical incision, reducing the duration of the procedure, and it is always under the surgeon's view. It can be cannulated directly or through a side graft sewn to the artery. The IA is often involved in TAAD, however, and thus cannot always serve as an arterial cannulation site. Similar outcomes and neuroprotection results for IA and
TABLE 2. Operations performed and perfusion data

\begin{tabular}{|c|c|}
\hline Variable & Value \\
\hline Ascending aorta replacement, $\mathrm{n}(\%)$ & $23(52)$ \\
\hline Bentall procedure, $\mathrm{n}(\%)$ & $10(23)$ \\
\hline Hemiarch replacement, n (\%) & $6(14)$ \\
\hline Total arch replacement, n (\%) & $5(11)$ \\
\hline \multicolumn{2}{|l|}{ Concomitant procedures, $\mathrm{n}(\%)$} \\
\hline AVR & $5(11)$ \\
\hline CABG & $2(5)$ \\
\hline $\mathrm{CPB}$ time, min, mean $\pm \mathrm{SD}$ & $185 \pm 62$ \\
\hline Cross-clamp time, min, mean $\pm \mathrm{SD}$ & $138 \pm 41$ \\
\hline ACP time, min, mean $\pm \mathrm{SD}$ & $44 \pm 15$ \\
\hline Lowest nasopharyngeal temperature, ${ }^{\circ} \mathrm{C}$, mean $\pm \mathrm{SD}$ & $24.6 \pm 1.3$ \\
\hline
\end{tabular}

axillary cannulation during surgery of the aortic arch have been reported. ${ }^{7}$

In January 2015, we started to cannulate the proximal portion of the right subclavian artery percutaneously with a single sternum access surgical incision extended to the right neck without a secondary infraclavicular incision for CBP and unilateral ACP in patients undergoing surgery of the thoracic aorta. This technique can be used also in TAAD because the right subclavian artery is less often involved in the dissection process compared with the IA. Thus also avoids the need for a timeconsuming secondary incision with its related complications.

The proximal portion of the right subclavian artery arises from the bifurcation of the right brachiocephalic trunk deep to the right sternocostal joint and is crossed by the vagus nerve. The right vagus descends between the internal jugular vein and the internal and common carotid arteries, then crosses anteriorly to the first part of the subclavian artery at the lower margin and gives off its right recurrent laryngeal branch surrounding the artery. Appropriate surgical exposure is mandatory to avoid the risk of vagus nerve injury.

In our study, no lesions to the vagus nerve or the recurrent laryngeal nerve were reported, and no vascular complications related to this technique were observed. No postoperative delayed gastric emptying as a consequence of vagus nerve injury was observed at clinical follow-up. No elevation of the right hemidiaphragm as a result of phrenic nerve injury was detected on postoperative chest X-ray and at discharge.

Our rates of operative mortality and neurologic adverse events were similar to those at other central cannulation sites reported by other groups. ${ }^{8,9}$ In our study, in-hospital mortality occurred in 3 patients suffering from TAAD, and permanent neurologic dysfunction occurred in 3 patients, 2 of whom presented with preoperative neurologic 


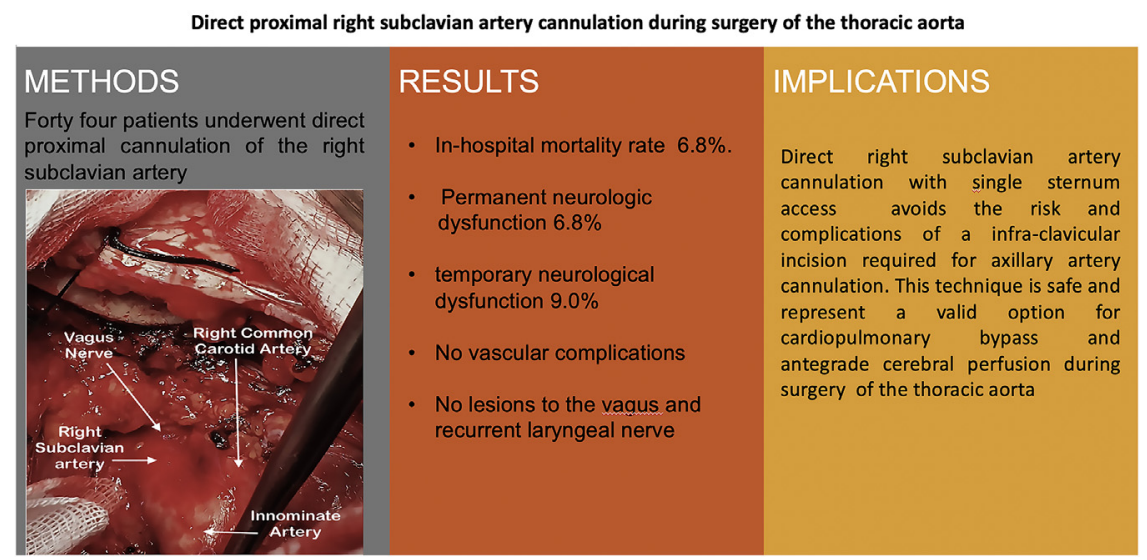

FIGURE 4. Direct proximal right subclavian artery cannulation can be performed with a single surgical incision via a median sternotomy. This technique is a safe and valid option for arterial cannulation during thoracic aorta surgery.

dysfunction. No patients died after elective surgery of the thoracic aorta.

Less invasive approaches for central cannulation sites in surgery of the thoracic aorta have been described. Garg and colleagues ${ }^{10}$ used the Seldinger technique to place a $14 \mathrm{Fr}$ catheter in the IA for ACP, but they used the distal aneurysmal aorta for arterial cannulation to establish CPB. Although their results were remarkable, this less invasive strategy cannot be safely adopted in TAAD. Moreover, 2 separated cannulae are required to achieve CBP and ACP. After distal anastomosis, the aortic line must be connected to the polyester graft to resume CBP and rewarming; thus, a side limb of the graft is needed to avoid bleeding from the cannulation site.

To avoid the risk of arterial injury and limb malperfusion that might occur after direct cannulation of the vessel and to reduce the more time-consuming sidearm anastomosis that often tends to bleed during CBP, Carino and colleagues ${ }^{11}$ used an open Seldinger-guided technique for axillary artery cannulation. Their approach minimizes the complications related to the small diameter of the vessel with excellent results; however, it still requires a secondary subclavicular surgical incision with all its drawbacks.

TABLE 3. Postoperative outcome

\begin{tabular}{lc}
\hline \multicolumn{1}{c}{ Outcome } & Number \\
\hline Hospital death & 3 \\
Reexploration for surgical bleeding & 3 \\
Respiratory failure* & 5 \\
Permanent neurologic dysfunction & 3 \\
Transient neurologic dysfunction & 4 \\
Paraplegia & 1 \\
Permanent hemodialysis & 2 \\
\hline
\end{tabular}

*Respiratory failure comprises postoperative pneumonia, pneumothorax, and tracheotomy.
Intrathoracic right subclavian artery cannulation without a secondary incision has been reported by Norton and colleagues. ${ }^{12}$ They described a series of 58 patients undergoing aortic surgery with right subclavian artery cannulation after full sternotomy by sewing an 8-mm Dacron graft to the artery close to its origin. In our study, the patients underwent percutaneous open Seldinger-guided right subclavian artery cannulation close to the origin of the right common carotid artery. This technique is faster than the side graft technique. The vessel is not clamped, reducing the risk of arterial and vagus nerve injury.

This study has several limitations, including the observational rather than comparative design, small sample size. It is a single-center-based study, limiting its generalization to other aortic surgery centers. Both the types and sites of cannulation were not chosen at random and were at the discretion of the surgeons in a heterogenous but consecutive group of patients. Although right subclavian artery cannulation is not currently accepted for aortic surgery worldwide, it could improve the surgical results and safely achieve ACP.

In conclusion, when feasible, an open Seldinger-guided technique for right subclavian artery cannulation is our preferred site for cannulation in surgery of the thoracic aorta and when the aortic dissection involves the IA. The technique is simple and fast and avoids the complications of a secondary infraclavicular incision. Contraindications are dissection extending distal to the right subclavian artery and small vessel diameter.

\section{Conflict of Interest Statement}

Dr Audo reported receiving funding from the private Uspitalet Foundation to provide supplies for the surgical ward. All other authors reported no conflicts of interest.

The Journal policy requires editors and reviewers to disclose conflicts of interest and to decline handling or reviewing manuscripts for which they may have a conflict 
of interest. The editors and reviewers of this article have no conflicts of interest.

\section{References}

1. Sabik JF, Nemeh H, Lytle BW, Blackstone EH, Gillinov AM, Rajeswaran J, et al. Cannulation of the axillary artery with a side graft reduces morbidity. Ann Thorac Surg. 2004;77:1315-20

2. Jassar AS, Vallabhajosyula P, Bavaria JE, Gutsche J, Desai ND, Williams ML, et al. Direct innominate artery cannulation: an alternate technique for antegrade cerebral perfusion during aortic hemiarch reconstruction. J Thorac Cardiovasc Surg. 2016;151:1073-8.

3. Schachner T, Nagiller J, Zimmer A, Laufer G, Bonatti J. Technical problems and complications of axillary artery cannulation. Eur J Cardiothorac Surg. 2005;27: 634-7.

4. Cavozza C, Campanella A, Pasquale P, Audo A. Percutaneous or side-arm graft right subclavian artery cannulation via median sternotomy. Aorta (Stamford). 2019;7:150-3.

5. Achneck HE, Rizzo JA, Tranquilli M, Elefteriades JA. Safety of thoracic aortic surgery in the present era. Ann Thorac Surg. 2007;84:1180-5.

6. Etz CD, von Aspern K, da Rocha E Silva J, Girrbach FF, Leontyev S, Luehr M, et al. Impact of perfusion strategy on outcome after repair for acute type A aortic dissection. Ann Thorac Surg. 2014;97:78-85.
7. Chu MW, Losenno KL, Gelinas JJ, Garg V, Dickson J, Harrington A, et al. Innominate and axillary cannulation in aortic arch surgery provide similar neuroprotection. Can J Cardiol. 2016;32:117-23.

8. Preventza O, Bakaeen FG, Stephens EH, Trocciola SM, de la Cruz KI, Coselli JS. Innominate artery cannulation: an alternative to femoral or axillary cannulation for arterial inflow in proximal aortic surgery. J Thorac Cardiovasc Surg. 2013; 145(3 Suppl):S191-6.

9. Strauch JT, Spielvogel D, Lauten A, Lansman SL, McMurtry K, Bodian CA, et al. Axillary artery cannulation: routine use in ascending aorta and aortic arch replacement. Ann Thorac Surg. 2004;78:103-8.

10. Garg V, Tsirigotis DN, Dickson J, Dalamagas C, Latter DA, Verma S, et al. Directinnominate artery cannulation for selective cerebral perfusion during deep hypothermic circulatory arrest in aortic surgery. J Thorac Cardiovasc Surg. 2014;148:2920-4.

11. Carino D, Mori M, Pang PYK, Singh M, Elkinany S, Tranquilli M, et al. Direct axillary cannulation with open Seldinger-guided technique: is it safe? Eur J Cardiothorac Surg. 2018;53:1279-81.

12. Norton E, Wu X, Makkinejad A, Farhat L, Yang B. Intrathoracic right subclavian cannulation in aortic arch surgery. Circulation. 2019;140:A15475.

Key Words: right subclavian artery, cannulation, arterial inflow, cardiopulmonary bypass 